\title{
Recommendations for patient screening in ultra-rare inherited metabolic diseases: what have we learned from Niemann-Pick disease type C?
}

María-Jesús Sobrido ${ }^{1 *}$, Peter Bauer²,3 ${ }^{2}$ Tom de Koning ${ }^{4}$, Thomas Klopstock ${ }^{5}$, Yann Nadjar ${ }^{6}$, Marc C Patterson ${ }^{7}$, Matthis Synofzik ${ }^{8,9}$ and Chris J Hendriksz ${ }^{10}$

\begin{abstract}
Background: Rare and ultra-rare diseases (URDs) are often chronic and life-threatening conditions that have a profound impact on sufferers and their families, but many are notoriously difficult to detect. Niemann-Pick disease type C (NP-C) serves to illustrate the challenges, benefits and pitfalls associated with screening for ultra-rare inborn errors of metabolism (IEMs).

A comprehensive, non-systematic review of published information from NP-C screening studies was conducted, focusing on diagnostic methods and study designs that have been employed to date. As a key part of this analysis, data from both successful studies (where cases were positively identified) and unsuccessful studies (where the chosen approach failed to identify any cases) were included alongside information from our own experiences gained from the planning and execution of screening for NP-C. On this basis, best-practice recommendations for ultra-rare IEM screening are provided. Twenty-six published screening studies were identified and categorised according to study design into four groups: 1) prospective patient cohort and family-based secondary screenings (18 studies); 2) analyses of archived 'biobank' materials (one study); 3) medical chart review and bioinformatics data mining (five studies); and 4) newborn screening (two studies). NPC1/NPC2 sequencing was the most common primary screening method (Sanger sequencing in eight studies and next-generation sequencing [gene panel or exome sequencing] in five studies), followed by biomarker analyses (usually oxysterols) and clinical surveillance.

Conclusions: Historically, screening for NP-C has been based on single-patient studies, small case series, and targeted cohorts, but the emergence of new diagnostic methods over the last 5-10 years has provided opportunities to screen for NP-C on a larger scale. Combining clinical, biomarker and genetic diagnostic methods represents the most effective way to identify NP-C cases, while reducing the likelihood of misdiagnosis. Our recommendations are intended as a guide for planning screening protocols for ultra-rare IEMs in general.
\end{abstract}

Keywords: Niemann-Pick disease, Screening, Diagnosis, Ultra-rare disease

\footnotetext{
*Correspondence: ssobrido@gmail.com

'Neurogenetics Research Group, Instituto de Investigación Sanitaria, Santiago

de Compostela, Spain

Full list of author information is available at the end of the article
}

(c) The Author(s). 2019 Open Access This article is distributed under the terms of the Creative Commons Attribution 4.0 International License (http://creativecommons.org/licenses/by/4.0/), which permits unrestricted use, distribution, and reproduction in any medium, provided you give appropriate credit to the original author(s) and the source, provide a link to the Creative Commons license, and indicate if changes were made. The Creative Commons Public Domain Dedication waiver (http://creativecommons.org/publicdomain/zero/1.0/) applies to the data made available in this article, unless otherwise stated. 


\section{Introduction}

Rare and ultra-rare diseases (URDs) are often chronic and life-threatening conditions that have a profound impact on sufferers and their families, but many are notoriously difficult to detect. Between 5000 and 8000 distinct rare diseases are documented (www.eurordis.org). Individually, these diseases are infrequent but collectively they affect 300 million people worldwide (www.eurordis.org) [1]. The definition of a URD varies based on different factors, including disease prevalence, symptom severity/impact, treatment availability, and heritability [2]. In the EU a URD is defined as affecting $<2: 100,000$ people $(<20$ patients per million) $[3,4]$. Inborn errors of metabolism (IEMs) represent a group of URDs collectively reported to affect up to $125: 100,000$ people $[5,6]$.

Ultra-rare IEMs have received increased attention in the last two decades due to the characterisation of causal genes and underlying metabolic pathways. This has enabled the development of targeted, disease-modifying treatments for a number of such conditions, including Niemann-Pick disease types A, B and C (NP-A/NP-B/ NP-C), Gaucher disease type 3 (GD3), Fabry disease, phenylketonuria (PKU), medium-chain acyl-CoA dehydrogenase deficiency (MCADD) and homocysteinemia, among others $[1,7,8]$. Such therapies can have a major effect on disease course, increasing patient quality of life and improving outcomes [9-11], but early and prompt initiation of treatment is usually required to minimise or prevent irreversible pathology (e.g., neuronal damage in neurodegenerative IEMs). Proactive strategies to enable timely diagnosis are therefore essential.

NP-C is an autosomal recessive, neurovisceral lysosomal storage disease (LSD) caused by mutations in the NPC1 or NPC2 genes (in $~ 95 \%$ and $\sim 5 \%$ of patients, respectively) $[11,12]$. These lead to impaired intracellular lipid trafficking and excess glycosphingolipid storage in various tissues including the brain and liver [13]. Affected patients exhibit highly heterogeneous clinical phenotypes involving progressive neurological and psychiatric manifestations as well as visceral symptoms [11]. The disease has pan-ethnic occurrence and has been estimated to affect at least 1:100,000 individuals $[1,12,14]$. However, it is believed that the true prevalence of the disease is higher, as cases can be masked by non-specific symptoms in certain clinical subpopulations [14, 15].

NP-C serves as a prime example to illustrate the challenges, benefits and pitfalls associated with screening for an ultra-rare IEM, since it has a number of features common to most such diseases [16]. It is a chronic, progressive condition involving high clinical heterogeneity and early mortality, and often goes undetected or misdiagnosed for prolonged periods due to non-specific manifestations. Diagnosis requires multidisciplinary work-up and multiple referrals to expert centres. There is limited awareness of symptoms suggestive of NP-C at the routine practice level, which can delay specialist referral and accurate diagnostics. Table 1 summarises the key features of NP-C alongside other IEMs with similar characteristics. All of them are URDs, with variable age at onset and heterogeneous clinical phenotype, almost invariably involving diverse neuropsychiatric manifestations.

The diagnosis of NP-C used to depend on time-consuming and costly laboratory techniques such as filipin staining and cholesterol esterification assays, with confirmatory Sanger genetic sequencing in single patients [10, 17-19]. However, increased knowledge of the disease has allowed the development of new screening and diagnostic methods. Simple clinical tools such as the NP-C suspicion Index (NP-C SI) help detect patients with a high likelihood of NP-C for further testing [2022]. Rapid, reliable and cost-effective blood biomarkers including oxysterols [23], lysosphingomyelins [24, 25], and bile acids are also now available $[18,26]$. In addition, powerful next-generation sequencing (NGS) methods, whole-exome sequencing (WES) and phenotype-specific gene panels can now be applied to entire patient cohorts as well as single patients [23, 26-28].

Disease screening can involve testing complete populations of asymptomatic individuals for the presence of certain disease markers. However, screening for ultra-rare IEMs on a population-wide basis is not generally considered appropriate due to a number of ethical, health economic, legal and regulatory limitations. Instead, ultra-rare IEMs are typically screened for through targeted testing of at-risk cohorts with certain relevant symptoms or risk factors. The WHO criteria for disease screening specify that new screening technologies must address a number of factors relevant to many URDs [29, 30]. An accepted treatment for the disease being screened must be available, the tests must be accessible, and the disease must feature a recognizable latent or early symptomatic stage, all of which are true for NP-C.

In this article, we review the wide range of methods and study designs that have been used to screen for NP-C, taking in lessons from both successful studies (where screening succeeded in identifying new cases) and unsuccessful studies (where the chosen approach failed to identify any cases). Specific 'diagnostic methods' include genetic testing, biomarker analysis, and clinically-based techniques. The term 'study design', as applied in our review, refers to overall screening approaches split into four categories: 1) prospective patient screening studies; 2) analyses of archived 'biobank' materials; 3) medical chart review and bioinformatics data mining; and 4) newborn screening. We reviewed the experience gained from the planning and execution of screening studies in NP-C as a representative example of an ultra-rare IEM. Finally, we propose 


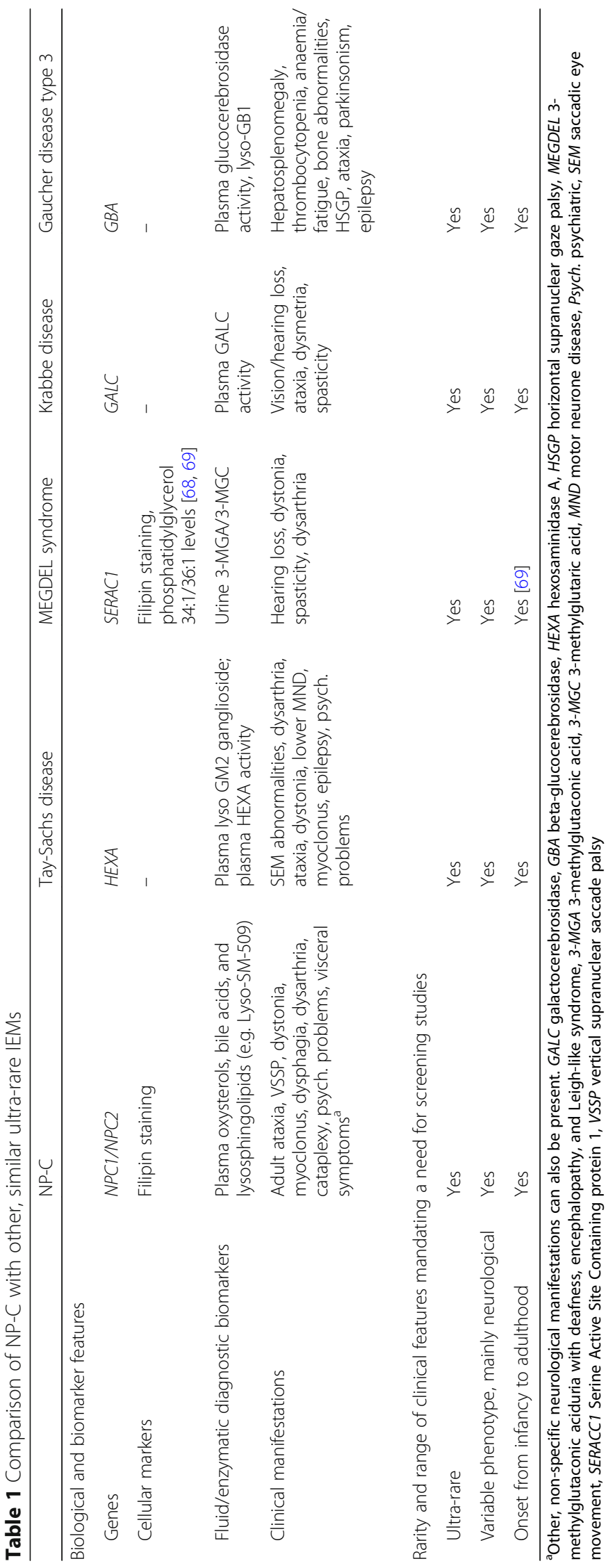


best practice recommendations that we believe could be extrapolated to screening protocols for other rare IEMs.

\section{Methodology}

A comprehensive, non-systematic review of published information was conducted using PubMed and Embase. All NP-C screening studies or studies in which NP-C was detected during screening of patients with unknown aetiologies were considered eligible. Articles published in English or at least with English abstracts between 2000 and 2018 were included. The main search terms were 'Niemann', 'screening' and/or 'diagnosis' (limited mainly to title/abstract fields). A pragmatic approach was adopted for the inclusion of articles due to the extremely varied nature of published literature relating to URD screening studies. No protocol for handling case redundancy between publications was included in the search since the emphasis of this review was on methodological approaches as opposed to establishing disease prevalence.

Each identified publication was examined to extract methodological features relating to: study population (e.g., population size, patient age, clinical phenotype); study type (e.g., observational or interventional, prospective or retrospective, controlled or not controlled/ naturalistic), diagnostic methods, study location (e.g., regional/international, single-centre/multicentre), medical specialty/disease area (e.g., neurology, paediatrics, hepatology, "any"), and inclusion of controls (e.g., healthy controls, disease-area controls). Available, unpublished methodological aspects of some of our own ongoing screening studies were also described, where relevant.

All identified studies were grouped in summary Table 2 based on overall study design (screening types). Further details of the included studies are provided in Additional files 1, 2 and 3 Table S1-S3, categorised by the primary diagnostic method. Many of the studies involved a combination of clinical, biochemical and genetic methods.

Findings from each published study were presented as the net number as well as the proportion (\%) of NP-C patients identified. Methodological details and relevant learnings from 'failed' studies, in which no NP-C patients were identified, were also considered. In addition, midto long-term 'halo' effects of screening studies were addressed in order to gauge any lasting impact due to increased awareness and implementation of new methods (e.g., subsequent inclusion of NP-C in local diagnostic protocols).

\section{Findings}

\section{Prospective patient screening studies}

Numerous prospective NP-C screening studies narrowed the screening focus by targeting cohorts with an increased disease risk, and found patients with NP-C who had previously gone undetected. Most such studies involved combinations of initial clinical assessments with one or both of genetic and biomarker analyses.

\section{Genetic screening}

Historically, the most widely used genetic analysis method for confirming a diagnosis of NP-C has been Sanger sequencing of NPC1 and NPC2 in individual patients with symptoms that are strongly suggestive of NP-C $[11,26]$. However, a number of studies also used this method to identify new cases within at-risk cohorts (Table 2; Additional file 1: Table S1). In a cohort of 250 adults with neuropsychiatric symptoms compatible with NP-C, Bauer et al. observed a higher incidence of NP-C $(1.2 \%)$ versus that in the general population (11.12:100,000 individuals (0.001\%)) [31]. In addition, 12 (4.8\%) heterozygous NP-C carriers (i.e., individuals with single $N P C 1 / N P C 2$ variants) were identified. NP-C cases have also been successfully identified using direct Sanger sequencing in patients with early-onset degenerative ataxia [32] and Huntington's disease-like manifestations (HD) [33].

Targeted Sanger-based screening of relatives following the diagnosis of probands with $N P C 1 / N P C 2$ variants confirmed a high prevalence of NP-C carriers in some regions. Based on a Turkish National Registration Database, Topcu et al. screened 510 family members of four NP-C probands with data suggestive of consanguinity. Two new NP-C patients $(0.4 \%)$ from two families were identified [34]. Notably, the overall frequency of heterozygous NPC1/NPC2 carriers in this cohort was $22.7 \%$.

Cohort studies have also been published in which no patients were diagnosed with NP-C using Sanger sequencing. Among 50 adults with early-onset neurodegenerative dementia and atypical symptoms ('dementia plus syndrome'), Cupidi et al. only observed four individuals with single $N P C 1$ or $N P C 2$ variants [35], and suggested a possible contributing role for $N P C 1 / N P C 2$ variants in these cases. In a large comparative cohort of patients with Parkinson's disease (PD), frontotemporal dementia (FTD) and progressive supranuclear palsy (PSP), Zech et al. reported identified only a single pathogenic NPC1/NPC2 variants in six patients $(1.1 \%)$, which did not differ significantly from the frequency of heterozygous variants in the general population [36].

Large NGS gene panels covering > 100 genes, WES and whole-genome sequencing (WGS) are becoming more manageable, accessible, and cost-effective [37, 38]. $N P C 1$ and NPC2 are currently included in gene panels for infantile cholestatic disease [39], early onset ataxia (EOA) [27], dystonia [38], IEMs [37], organic psychosis, early-onset cognitive decline, hepatosplenomegaly, and developmental delay. A number of studies have reported the successful use of NGS-based methods in identifying 
Table 2 Summary of published screening studies grouped by screening design

\begin{tabular}{|c|c|c|c|}
\hline Cohort (reference) & Study population & $N$ & Region/count \\
\hline \multicolumn{4}{|c|}{ Prospective patient screening studies } \\
\hline \multicolumn{4}{|l|}{ Genetic screening } \\
\hline Bauer et al. 2013 [31] & Adults with neurological/psych. Symptoms & 250 & EU and US \\
\hline Schicks et al. 2013 [32] & $\begin{array}{l}\text { Adults with ataxia, EOCD and suspected } \\
\text { recessive disease }\end{array}$ & 24 & Germany \\
\hline Zech et al. 2013 [36] & Adults with PD, FTD or PSP & 790 & Germany \\
\hline Nanetti et al. 2017 [33] & Adults with suspected HD & 18 & Italy \\
\hline Topcu et al. 2017 [34] & Family members of NPC1/NPC2 probands & 510 & Turkey \\
\hline Cupidi et al. 2017 [35] & Adults with early-onset 'dementia-plus' & 50 & Italy \\
\hline Synofzik et al. 2015 [27] & Adolescents/adults with unexplained EOA & 96 & Germany \\
\hline Marelli et al. 2016 [40] & Adolescents/adults with probable EOA & 33 & France \\
\hline Pyle et al. 2015 [41] & $\begin{array}{l}\text { Adult patients with inherited and sporadic } \\
\text { ataxias }\end{array}$ & 35 & UK \\
\hline McKay et al. 2014 [42] & Infants with jaundice/cholestasis & 228 & UK \\
\hline Herbst et al. 2015 [43] & Infants with jaundice/cholestasis & 6 & Germany \\
\hline Mavridou et al. 2014 [70] & Family members of NP-C patients & 153 & Greece \\
\hline Wassif et al. 2016 [14] & Subjects from 4 WES sequencing projects & $17,754^{c}$ & International \\
\hline
\end{tabular}

\section{Blood biomarker screening}

Reunert et al. 2016 [44]

Ribas et al. 2016 [45]

Zhang et al. 2014 [46]

De Castro et al. 2017 [47]

Sheth et al. 2014 [48]
Patients with suspected NP-C

Patients with suspected NP-C

Children/adults with cholestasis, HSL or psychomotor regression/retardation

Patients with suspected NP-C

Children with possible LSDs

$\begin{array}{ll}1800 & \text { Germany } \\ 122 & \text { Brazil } \\ 302 & \text { China } \\ 236 & \text { Spain }\end{array}$

1110

India, Sri Lanka, Afghanistan

Spain

US

Hegarty et al. 2015 [52]

Verity et al. 2010 [53]

Winstone et al. 2017 [54]

Corry 2014 [55]

$\begin{array}{lll}97 & \text { Spain } & \text { Oxysterol level (7-KC) } \\ 40 & \text { US } & \text { Medical chart review } \\ 127 & \text { UK } & \begin{array}{l}\text { Clinical, laboratory, and } \\ \text { outcome analysis }\end{array} \\ 2636 & \text { UK } & \text { Clinical case surveillance } \\ 3979 & \text { UK } & \text { Clinical case surveillance } \\ 13,000 & \text { UK } & \text { Clinical case surveillance }\end{array}$

Patients with NP-C

Neonates with jaundice/cholestasis

Children with acute liver failure

Children with early cognitive impairment

Children with intellectual and neurological deterioration

Ethnic subjects with suspected autosomal recessive conditions
Studies based on newborn screening

Pinto et al. $2004[60]$

Antenatal patients with suspected LSDs

353

Portugal

NPC1/NPC2 sequencing ${ }^{a}$

Polo et al. 2016 [61]

Neonates with cholestasis
$7 \quad$ Italy

Oxysterols (7-KC, C-triol)

${ }^{\mathrm{a}}$ Sanger sequencing; ${ }^{\mathrm{b}}$ Mini-exome sequencing of 5,000 genes; ${ }^{C} \mathrm{WES}$ of 17,754 chromosomes. ChT chitotriosidase, CNV copy-number variation, EOA early-onset ataxia, EOCD early-onset cognitive decline, FTD frontotemporal dementia, GAG glycosaminoglycans, HRM high resolution melting, HSL hepatosplenomegaly, LSD lysosomal storage disease, NGS next-generation sequencing, PD Parkinson's disease, PSP progressive supranuclear gaze palsy, Psych. psychiatric, RFLP restriction fragment length polymorphism, WES whole-exome sequencing 
previously undiagnosed NP-C cases in at-risk cohorts, particularly among patients with cerebellar ataxia of unclear origin - an extremely heterogeneous clinical population in which genetic diagnoses are notoriously difficult to achieve (Table 2; Additional file 1: Table S1). In a study of 96 patients with unexplained EOA (age at onset $<40$ years), targeted high-throughput sequencing of 122 known ataxia genes including NPC1 and NPC2, $\mathrm{NP}-\mathrm{C}$ diagnoses were confirmed in $2 / 96$ patients $(2.1 \%)$ [27]. The total frequency of NPC1/NPC2 gene variants was $8 / 192$ (4.2\%), indicating an enrichment of rare $N P C 1 / N P C 2$ variants in EOA subjects compared with the general population $(203 / 12,962(1.6 \%))$. Another study found two (6.1\%) NP-C cases in 33 patients with suspected inherited ataxia (age at onset $<50$ years) using mini-exome and copy-number variation (CNV) analysis [40]. Using WES, Pyle et al. reported two siblings (5.7\%) with NP-C among 22 randomly selected families affected by unexplained ataxias [41]. Castro-Fernández and colleagues identified three patients with previously undiagnosed NP-C among 26 adults with progressive ataxia and other movement disorders, using targeted gene panel sequencing (Sobrido MJ, personal communication).

Liver disease is common early in the course of NP-C, and cohorts of young patients have been assessed using NGS to rule out genetic causes of infantile cholestasis. In independent studies of such patients, McKay et al. [42] and Herbst et al. [43] diagnosed NP-C in 1/228 (0.4\%) and $1 / 6(16.7 \%)$ subjects using custom-designed gene panels targeting NPC1/NPC2 alongside other genes associated with cholestatic disease in infancy and childhood.

\section{Blood biomarker screening}

Plasma oxysterol assays are now available in over 30 laboratories worldwide, and findings from their use have been reported in a number of screening studies (Table 2; Additional file 2: Table S2). Plasma lysosphingolipid and bile acid assays are relatively new and have the advantage of being detectable in dried blood spots (DBS). However, to date, there are no published reports on their use in NP-C screening.

Two prospective studies that included patients with clinical suspicion of NP-C and which used the oxysterol biomarker, cholestane- $3 \beta, 5 \alpha, 6 \beta$-triol (C-triol), provided NP-C detection rates of $4.0 \%$ [44] and $9.8 \%$ [45]. In a further cohort study of patients referred for either cholestasis/hepatosplenomegaly/isolated splenomegaly, or psychomotor regression/retardation, Zhang et al. diagnosed NP-C in 4.0\% of patients based on elevated plasma levels of another oxysterol, 7-ketocholesterol (7-KC) [46]. In all three studies, diagnoses were confirmed by genetic analysis of NPC1/ NPC2 mutations.

Other biomarker methods have been variably applied to screen patient cohorts for NP-C. In 236 patients with clinical suspicion of NP-C, De Castro et al. [47] diagnosed 10 patients $(4.2 \%)$ based on plasma chitotriosidase (ChT) and $\mathrm{C}-\mathrm{C}$ motif chemokine ligand 18 (CCL18/PARC) levels alongside NP-C SI assessments. Three further NP-C cases were identified in subsequent evaluations of patient family members. In another study of children referred for metabolic testing due to symptoms suggestive of LSDs, Sheth et al. [48] reported four NP-C patients $(0.1 \%)$ based on filipin staining of cultured fibroblasts. A screening study of 83 patients with unclassified cognitive impairment did not report any NP-C case based on plasma biomarkers (ChT and C-triol), clinical symptoms and NP-C SI [49].

Finally, findings are pending from a further screening study in adults with a first episode of acute psychosis based on a panel of biomarkers and metabolites, where included patients are being screened for a range of IEMs and immunological disorders (CJ Hendriksz, personal communication).

\section{Studies based on archived (biobank) samples}

Biobank studies involve the analysis of historical/archived blood, tissue or genetic materials. Currently there are no published biobank-based screening studies on NP-C, but reports of this study type are expected in the future. Cebolla et al. reported the use of archived biobank plasma samples to evaluate the utility of plasma 7-KC, ChT and CCL18/PARC in 97 patients with NP-C versus a number of control groups [50]. Plasma 7-KC concentration allowed discrimination between NP-C patients, NP-C carriers, and GD patients, but not from patients with NPA/B. Of note, plasma 7-KC and CCL18/PARC in patients with high NP-C SI scores were considered more useful than other biomarkers for defining which patients should undergo confirmatory genetic testing.

\section{Studies based on medical chart review and bioinformatics/data mining}

Screening studies based on patient file and clinical chart review have been conducted in order to detect new $\mathrm{NP}-\mathrm{C}$ cases as well as to estimate the incidence of NP-C (Table 2; Additional file 3: Table S3). Yerushalmi et al. reviewed clinical and laboratory information from 40 neonates with cholestasis at a paediatric liver centre [51]. Clinical chart review and confirmatory cholesterol esterification assays, liver lipid measurements and genetic analyses identified NP-C in three babies (7.5\%) who were initially thought to have idiopathic neonatal hepatitis. Hegarty et al. analysed historical data from clinical and laboratory assessments in 127 newborns and infants with acute liver failure [52], and diagnosed three NP-C patients $(2.4 \%)$ out of a total of $36(28.3 \%)$ who had a confirmed metabolic aetiology. 
Historical health surveillance data can also be accessed to screen for rare conditions. A study from the British National Surveillance Unit (BPSU) provided cross-sectional data on the occurrence of rare disorders including variant Creutzfeldt-Jacob syndrome (vCJD) and NP-C as underlying causes of progressive intellectual and neurological deterioration (PIND) [53, 54]. Over 12 years, 2636 patients aged $<16$ years were sent for further assessment of underlying rare disorders, and subsequent expert review of anonymised patient records reached diagnoses that explained observed symptoms in 1114 patients (42\%). Among those, NP-C was detected in 38 patients (1.4\%). A 2017 update of the study supported the original detection rate (1.3\%) [54], and the lifetime risk of NP-C as a cause of PIND among children was estimated at 0.38 per 100,000 live births. Notably, both of these studies highlighted high rates of PIND in areas with higher rates of consanguinity - a recognised predisposing factor in NP-C [15].

Data mining studies, where pre-existing databases are examined to generate new data, represent another form of retrospective, file-based patient screening. A UK study compiled information from regional and national patient registries, reporting a higher prevalence of autosomal recessive conditions (including NP-C) in an ethnic subpopulation $(N=13,000)$ versus the general population [55]. Similar to the BPSU health surveillance studies [53, 54], this finding served to highlight the influence of consanguinity/endogamy on the prevalence of autosomal recessive diseases in some UK communities.

A data mining project employing a bioinformatics methodology is currently underway in Germany. This project, called "mineRARE", aims to identify patients with rare disorders (including NP-C) by using semantic text-mining of electronic medical records. Results are currently pending ( $\mathrm{T}$ Klopstock, personal communication).

\section{Studies based on newborn screening}

Newborn screening in ultra-rare IEMs, particularly those associated with late-onset symptoms, requires consideration of a number of ethical, clinical, legal and cultural issues $[56,57]$. NP-C is not currently included in routine newborn screening programs due to: 1 ) the vast heterogeneity of clinical manifestations and prognosis; 2) the nature of therapeutic benefits achievable with therapy [57]; and 3) the fact that in many patients (20-30\%), symptom onset occurs relatively late in life [58, 59]. Hence, here we use the term 'newborn screening' to indicate screening in neonates with clinical abnormalities indicating possible NP-C or other URDs (e.g., perinatal liver disease), and in patients from at-risk clinical groups who did not have observable abnormalities. As such, newborn screening for NP-C can be considered a special subtype of prospective screening studies.
Two studies have investigated the utility of newborn screening for LSDs in general, or NP-C specifically. Pinto et al. reported a 20-year retrospective analysis of 29 different LSDs at a reference centre for antenatal diagnosis [60]. A total of 353 LSD patients were identified out of 4700 cases, among whom 18 patients $(0.4 \%)$ were diagnosed with NP-C. Based on these results the birth prevalence of NP-C was estimated at 2.2 cases per 100,000 . Using oxysterol measures as a screening approach, Polo et al. reported substantially increased levels of both $\mathrm{C}$-triol and 7 -KC in 6 out of 7 neonates from a selected cohort with severe cholestasis and suspicion of NP-C [61]. However, genetic testing confirmed a diagnosis of NP-C in only one of these. The observed high rate of false-positives in this cohort was considered a potential pitfall of oxysterol analysis as a screening tool in cholestatic neonates.

\section{Recommendations on screening set-up for ultra- rare IEMs}

There is significant overlap between disease features of NP-C and other ultra-rare IEMs, and similar challenges are faced upon screening for these diseases. Based on published NP-C screening studies and our own experiences, we identified key issues related with likelihood of successful screening and developed a set of recommendations for the setup of screening studies in ultra-rare IEMs (Table 3). General guidelines and local, national and international requirements for good practice in clinical studies also need to be considered.

The main objectives of ultra-rare IEM screening programs are to: 1) identify patients who would otherwise go undetected or receive a delayed diagnosis and thus go deprived of proper treatment; 2) characterize the phenotypic range where current suspicion is only based on a classical clinical syndrome; 3) evaluate gene variants as possible contributors to other diseases; and, 4) improve disease awareness to ensure inclusion of rare disorders in differential diagnosis. Additional deliverables from screening for ultra-rare IEMs include; assessment of gene variant effects in heterozygotes (carriers); identification of other unrecognised diseases during differential diagnosis; estimation of disease prevalence and incidence.

\section{Study design}

Key factors that should be addressed in designing a screening study include: 1) identification and selection of an appropriate patient cohort based on available data (e.g., from living patients, biobank samples, medical charts); 2) the use of optimal and accepted diagnostic method(s) (see Diagnostic methods); and 3) relevant local factors (e.g., available expertise, funding, regulations).

- Consider which at-risk patient populations might include 'hidden' ultra-rare IEM patients. 
Table 3 Key factors influencing success of screening studies for ultra-rare IEMs

\begin{tabular}{ll}
\hline Factor & Recommendation \\
\hline Team & Ensure patient detection and data quality through use of multidisciplinary investigator teams \\
Cohort size & Bear in mind that larger cohorts, possibly recruited via expert consortia/registries in at-risk cohorts, help capture \\
& the full phenotype range and prevalence data \\
Inclusion & Consider the impact of inclusion criteria that are neither too restrictive nor too broad \\
Methods & Employ methods based on associated advantages/limitations, minimally invasive sampling, formal requirements, \\
and possible confounding factors & Consider that large NGS gene panels/WES allow screening for multiple diseases in whole cohorts, and factor in \\
the sensitivity and specificity of genetic profiling methods & Choose biomarkers bearing in mind their sensitivity, specificity, validation, sample stability and ease of transport, \\
Biomarkers & and assay turnaround times \\
Clinical assessment & Use available simple clinical tools that allow quick analyses of relevant symptom clusters \\
Laboratories & Select reference laboratories with well-established infrastructure for selected, validated diagnostic method(s) \\
Consent & Take patient consent limits into account, particularly for retrospective chart reviews/biobanks \\
Sustainability & Preserve awareness and knowledge from screening studies in local diagnostic procedures and/or follow-up \\
processes & Raise awareness of rare disorders as a group represent a significant healthcare problem: this can aid referral \\
Increased awareness & to appropriate specialist clinics in time
\end{tabular}

- Define simple, concise screening objectives addressing appropriate clinical disease phenotypes.

- Involve the lay-community through medical education on IEM natural history (as for NP-C).

\section{Prospective study designs}

Prospective studies have the advantage of allowing further examination in suspected patients. However, in the case of ultra-rare IEMs, prospective studies can pose great challenges for patient recruitment and/or require prolonged observation periods in order to confirm a diagnosis.

\section{Retrospective study designs}

Retrospective studies are more suited to patient chart reviews and biobank analyses, and generally have simpler requirements versus prospective studies regarding logistics and planning. However, retrospective studies in ultra-rare IEMs may be prone to bias due to limited patient followup. Retrospective studies also depend on analyte stability, and expiration of patient consent may be a limiting issue. Limitations on data accuracy/completeness, potential for recall bias, and existence of missing data can be encountered in medical chart reviews. Access to corresponding physicians and/or patients (e.g., outdated contact details, patient death, physician retirement) also affect findings when older files or biobank samples are included. Biobanks must allow proper pre-selection of at-risk patients. Care must be taken to avoid over-interpretation of retrospective data, especially when information at hand is incomplete.

\section{Patient population}

Direct access to target screening population must be ensured.

- Effective collaboration between general physicians and expert centres is crucial in ultra-rare IEM screening programs, as general physicians are usually closer to the patients and their main healthcare reference.

- Common scenarios for patient sourcing include: atrisk cohorts in patients with key ultra-rare IEM symptoms; verification of published serendipitous findings in specific patient subgroups; patients considered at risk for scientific reasons (e.g., similar brain pathology in in neurodegenerative disorders).

\section{Cohort size}

Formal guidance on appropriate cohort sizes is lacking for many diseases, particularly ultra-rare IEMs. Target patient numbers should be addressed in a pragmatic manner according to the study design, diagnostic methods and epidemiological information. Larger screening cohorts potentially capture more disease phenotypes and provide more accurate prevalence estimates, but demand more resources and bear a higher chance of false positives.

- The number of potential patients affected by an ultra-rare IEM is very small. Hence, studies may need to include multiple centres or involve pertinent disease consortia or registries (e.g., the autosomalrecessive ataxia consortium, 'PREPARE' and the EOA registry in the case of $\mathrm{NP}-\mathrm{C}$ ). 
- The availability of historical data should be considered in studies aiming to estimate disease prevalence or incidence.

- Relevant age groups/disease stages are important where early identification is required in an ultra-rare IEM.

- Endogamy and consanguinity must be considered when studying IEMs in certain regions.

\section{Inclusion/exclusion criteria}

Clear and easy-to-follow inclusion/exclusion criteria should be defined that meet the consensus of the scientific community. The restrictiveness of chosen criteria influences detection accuracy: broader inclusion typically results in low detection rates, whereas more stringent criteria give higher detection rates. While this may seem obvious it has a particularly high impact in ultra-rare IEMs.

- Cohort inclusion and exclusion criteria (e.g., based on symptom severity or comorbidities) should be defined clearly for specific at-risk patient groups in ultra-rare IEMs that feature high phenotypic heterogeneity.

- Overly specific criteria might miss mild/atypical patients, which are common in ultra-rare IEMs.

- Overly specific criteria might miss patients with mild/atypical symptoms, which are common in ultra-rare IEMs. Furthermore, a bias toward subjects with classical disease presentations is likely present in the published literature, and thus the full phenotypic spectrum of rare disorders may not be well known.

\section{Diagnostic methods}

Screening methods for inherited disorders typically include clinical assessments of specific disease symptoms, biomarkers, and genetic tests. All three of these methods have utility for the detection of patients when applied on a broad scale. Taking NP-C as an example, key features of these methods are summarised in Table 4.

- Multi-analyte MS/MS biomarker panels or large NGS gene panels/WES allow cost-effective, simultaneous screening for diseases associated with clinical features that are common within a chosen screening cohort: such techniques can currently be applied in DBS samples for over 30 IEMs and are of particular use in newborn screening.

- DBS samples are particularly convenient in terms of storage and transport.

- Gene panels should cover all known diseases that can cause the same manifestations.
The most appropriate diagnostic methods should be chosen based on the following criteria:

1) Quality: the sensitivity, specificity, validity and robustness of the chosen test(s), and methods for identifying false positives and false negatives should be ensured. Methods should be acknowledged by the scientific community and acceptable to patients: the less invasive the better. Diagnostic methods in ultra-rare IEMs are not always supported by substantial published evidence, but may nevertheless be accepted by experts.

2) Suitability: diagnostic reference laboratories need to be experienced with the selected diagnostic method(s), and local infrastructure should grant access to IEM patients and sample shipment in less densely-populated areas.

3) Applicability and ease of use: processing limitations can prevent use of certain methods in some geographical areas, and possible confounding factors (e.g., auto-oxidation in plasma samples) should be taken into account. The effects of local cultural factors on patient agreement to participate must be considered in ultra-rare IEMs: the genetic nature of these disorders requires DNA analysis. The supply of relevant clinical background for less well known ultra-rare IEMs is vital to help diagnostic laboratories interpret findings.

\section{Multi-level diagnostic approaches}

Combinations of diagnostic methods including clinical assessments, biomarker assays, and/or genetic techniques can reduce the likelihood of screening errors, which is important in uncertain cases, as often seen in ultra-rare IEMs.

- Clinical tools assessing relevant symptom clusters can help distinguish affected patients from the general clinical population and non-affected patients.

- Biomarker analyses typically include confirmation of initial biomarker-identified cases though genetic analysis.

- Genetic screening studies are usually more successful when performed on patients/cohorts that have been selected through clinical assessments and/ or biomarker analysis.

\section{Ethical and regulatory requirements}

As in any disease, ethical and regulatory requirements must always be met in ultra-rare IEM screening, and include specific institutional/regulatory ethical approvals, regulatory body expectations, patient consent requirements, Good Clinical Practice (ICH-GCP) standards, and Good Laboratory Practice criteria. 
Table 4 Key features of diagnostic methods for ultra-rare IEMs: NP-C as an example

\begin{tabular}{|c|c|c|}
\hline Method & Examples for NP-C & Key features \\
\hline \multirow[t]{8}{*}{ Biomarkers } & - Oxysterols (C-triol, 7-KC) & - Advantages \\
\hline & \multirow[t]{2}{*}{ - Lysosphingolipids (Lyso-SM-509, lysosphingomyelin) } & - Objective, quantitative methodology \\
\hline & & - Rapid, practical and cost-effective* \\
\hline & \multirow[t]{5}{*}{ - Bile acids (3ß,5a,6ß-trihydroxycholanic acid) } & - Biomaterials easily accessible and transportable \\
\hline & & - Disadvantages \\
\hline & & - Available for relatively few ultra-rare IEMs \\
\hline & & - Requires that disease of interest is already in differential diagnosis \\
\hline & & $\begin{array}{l}\text { - Patient heterogeneity can present a hurdle, with possible } \\
\text { false-negatives/positives }\end{array}$ \\
\hline \multirow[t]{10}{*}{ Genetic analysis } & - Single-gene sequencing & - Advantages \\
\hline & - Gene panel (e.g., ataxia panel) & - Objective screening data \\
\hline & $\cdot$ WES & - No requirement for differential diagnosis \\
\hline & \multirow[t]{7}{*}{$\cdot$ WGS } & - Can provide information on diseases not in differential diagnosis \\
\hline & & - Might indicate alternate molecular diagnosis \\
\hline & & - Disadvantages \\
\hline & & - Not yet widely available without appreciable costs \\
\hline & & $\begin{array}{l}\text { - Limited information on pathogenicity of unique gene variants } \\
\text { (potential false negatives and false positives) }\end{array}$ \\
\hline & & $\begin{array}{l}\text { - Challenging management of VUS in symptomatic patients without } \\
\text { biochemical marker findings }\end{array}$ \\
\hline & & - Management of incidental findings \\
\hline \multirow[t]{9}{*}{ Clinical assessment } & - Multi-disciplinary assessment of clinical manifestations & - Advantages \\
\hline & - Differential diagnosis & $\begin{array}{l}\text { - Widespread availability of professionals capable of carrying out } \\
\text { clinical assessment }\end{array}$ \\
\hline & \multirow[t]{3}{*}{ - Assessment of clinical picture from patient files } & - Traditional approach set up in healthcare systems \\
\hline & & - Disadvantages \\
\hline & & - Can be time consuming \\
\hline & \multirow[t]{4}{*}{$\cdot N P-C S I$} & - Require multiple inter-disciplinary referrals \\
\hline & & $\begin{array}{l}\text { - Variation in quality: assessments not based on validated clinical } \\
\text { tools require IEM expert knowledge to detect disease }\end{array}$ \\
\hline & & $\begin{array}{l}\text { - Non detection of atypical/non-standard or early-stage presentations } \\
\text { due to non-specific clinical phenotypes }\end{array}$ \\
\hline & & $\begin{array}{l}\text { - Do not deliver diagnosis per se although diagnoses can be } \\
\text { confirmed using biomarkers and/or genetic methods }\end{array}$ \\
\hline
\end{tabular}

${ }^{a}$ Cost effectiveness depending on local infrastructure and/or geographical region; 7-KC 7-ketocholesterol, C-triol cholestane-3ß,5a,63-triol, NP-C SI NP-C suspicion index, VUS variant of unknown significance, WES whole-exome sequencing, WGS whole-genome sequencing

- Control of patients' personal information is particularly important in ultra-rare IEMs as patients can more easily be identified based on relatively few generic personal data. Thorough data anonymization should be implemented.

- Screening studies for diseases with available targeted therapies should be given priority.

\section{Screening logistics}

Logistics for sample handling, labelling, stability, and transport are crucial in ultra-rare IEMs as diagnostic tests are frequently carried out in specialist laboratories that may not be local. Definition of all aspects of sample storage is also important in biobank-based studies.

\section{Study team and disease experience}

Ultra-rare IEM screening studies are usually conducted by physicians with access to relevant cohorts but not necessarily with relevant expertise. Referring physicians should be well instructed and trained on the key disease signs and symptoms of ultra-rare IEMs. 
- Broad experience within the study team is vital: patient detection and data quality is best optimized in a multidisciplinary setup.

\section{Study legacy or 'halo' effects}

Potential long-term post-study benefits ('halo' effects) should be considered before starting a screening study, especially with less well recognized diseases like ultra-rare IEMs. Examples include: establishment of collaborative structures and improved lines of referral; creation of multi-analyte biomarker or gene panels that can be included in routine practice; and enduring local use of diagnostic methods/algorithms.

- Some ultra-rare IEM screening studies identify very few or no patients during the study observation period but cases can be identified subsequently due to increased local awareness, health provider acceptance of new biomarkers, and establishment of multidisciplinary care networks.

- Raised awareness is a potent factor in considering IEM diagnoses in some cohorts, and has been shown to ease acceptance of biomarker methods.

\section{Possible future screening strategies in NP-C}

A large proportion of published NP-C screening studies have employed combinations of both established and new diagnostic methods. Such strategies may reduce the likelihood of screening errors in the future. A typical diagnostic tactic for NP-C screening would comprise initial clinical examination (e.g., using the NP-C SI) followed by biomarker measurements and genetic validation. Combined approaches like this limit burden to patients and allow a more efficient and cost-saving study set-up [26, 47].

The general consensus among experts involved in NP-C care is that genetic analysis is mandatory for the confirmation of diagnosis [62]. New, rapid genetic sequencing methods such as WES and WGS are likely to allow wider screening across known at-risk patient cohorts in the near future. The potential application of NGS methods as the initial (first-line) diagnostic test in an ultra-rare IEM depends on available resources, genetic mutation types and complexity, disease awareness, and the nature of the disease and patients/cohorts in question. For instance, genetic analysis of FMR1 variants would not work as a screening method for Fragile X syndrome. Nevertheless, based on experience to date in NP-C, the potential inclusion of ultra-rare IEM genes in large NGS gene panels holds great promise for future screening protocols. The use of WES and WGS databases is growing, and ultra-rare IEM gene databases are increasingly becoming interconnected and/or made public. Where possible, an 'exome-first' approach, where WES is conducted as a first step to identify potential new cases in at-risk cohorts, may provide a more direct route to NP-C diagnosis [63]. Such approaches are already being implemented in some centres.

Updated international recommendations for the diagnosis and screening of NP-C classify new biomarker assays alongside genetic analyses as first-line diagnostic methods, and note that most diagnoses can be confirmed by the combined use of these methods [23]. The prospect of automatically linking large registries for at-risk clinical cohorts to relevant biomarker analyses is an interesting prospect for improving the detection of further cases, but is currently only applicable in the academic research setting. Such an approach is currently being assessed for linking lysosphingolipid assays with the EOA Registry in Germany (M Synofzik, personal communication).

\section{Conclusions}

Screening studies in NP-C, which is considered as a suitable role model for ultra-rare IEMs in general, are associated with a number of challenges related to the ultra-rare nature of the disease. To date, screening for NP-C has largely been based on single-patient studies, small case series, and targeted cohort studies in at-risk patient groups. However, the emergence of new diagnostic methods over the last 5-10 years has provided opportunities to screen for NP-C on a larger scale in whole at-risk cohorts [64-66].

NP-C is difficult to detect using routine methods as it is a lysosomal disorder that is not detected by standard enzyme panels. The advent of readily available, specific blood biomarkers has largely overcome this limitation, and the inclusion of specific biomarker assays into metabolic screening panels that can easily be applied in suspected patients or cohorts is now achievable. At a number of centres, biomarker assays have been used as a first-line step in diagnosing NP-C, allowing an exponential increase in the number of patients that can be screened in a short time [23, 26]. However, the position of biomarker testing in the diagnostic pathway varies between centres.

Broad genetic screening of patients with symptoms of unclear origin using NGS gene panels can now be conducted in large patient populations as well as in individual patients with no clear molecular diagnosis, and the use of NGS is expected to grow significantly in the future. Gene panels allow diagnostic testing for multiple ultra-rare IEMs. A growing number of centres are adopting an 'exome-first' diagnostic work-up in their routine practice (e.g. in Nijmegen, the Netherlands and Tübingen, Germany), whereby WES is applied widely before more detailed laboratory work-up.

New, simple digital clinical screening tools that allow rapid analyses of relevant symptom clusters are increasingly 
becoming available $[15,20]$. As an example, the NP-C SI allows rapid appraisal of the likelihood of NP-C at initial patient presentation or soon after, and helps to direct further, more detailed confirmatory tests.

Combining clinical, biomarker and genetic diagnostic methods represents the most effective way to identify new NP-C cases. Updated diagnostic and screening recommendations for NP-C have been developed that cover all available diagnostic methods, and should be considered when formulating any new screening study $[11,23]$.

Overall, the value of screening for ultra-rare IEMs such as NP-C represents a trade-off between funding costs on one hand, and benefits from targeted therapy in what are usually quite small yields of previously unidentified patients on the other [29]. In terms of cost-effectiveness, two types of study design can now be considered: a) based on gene panels and/or multi-analyte biomarker panels, which is associated with higher initial costs but can cover a large number of diseases [27, 42, 43]; and b) using relatively low-cost plasma- or DBS-based biomarkers that cover only single or a few diseases [67]. There is also an asymmetry in the number of available studies and resources devoted to disease screening for different IEMs based on the commercially-funded availability of targeted therapies. While this might potentially introduce some bias to reported case identification, this should not impede analysis of the existing literature and extraction of useful lessons.

Many of the learnings from NP-C screening studies can be extrapolated to other ultra-rare IEMs due to similarities in a number of key disease factors. These recommendations can therefore serve as a guide for planning patient screenings in ultra-rare IEMs in general.

\section{Additional files}

Additional file 1: Summary of published screening studies based primarily on genetic analysis. (DOCX $27 \mathrm{~kb}$ )

Additional file 2: Summary of published screening studies based primarily on biomarker analysis. (DOCX $29 \mathrm{~kb}$ )

Additional file 3: Summary of published screening studies based primarily on medical chart review and bioinformatics/data mining. (DOCX $29 \mathrm{~kb})$

\footnotetext{
Abbreviations

7-KC: 7-ketocholesterol; ChT: Chitotriosidase; CNS: Central nervous system; Ctriol: Cholestane-3 $\beta, 5 a, 6 \beta$-triol; DBS: Dried blood spot; EOA: Early-onset ataxia; GC/MS: Gas chromatography-mass spectrometry; GD: Gaucher disease; IEMs: Inborn errors of metabolism; LSD: Iysosomal storage disease; MCADD: Medium-chain acyl-CoA dehydrogenase deficiency; MEGDEL: 3methylglutaconic aciduria with deafness, encephalopathy and Leigh-like; MPS: Mucopolysaccharidoses; NP-A/NP-B: Niemann-Pick types A and B; NP-C SI: NP-C suspicion Index; NP-C: Niemann-Pick disease type C;

PCR: Polymerase chain reaction; PIND: Progressive intellectual and neurological deterioration; PKU: Phenylketonuria; URDs: Ultra-rare diseases; vCJD: variant Creutzfeldt-Jacob syndrome.
}

\section{Acknowledgements}

Matthew Reilly PhD provided medical writing support in the preparation of this manuscript, paid for by Actelion Pharmaceuticals Ltd. Matthis Synofzik is supported by the Else-Kröner Fresenius Stiftung and the grant 779257

"Solve-RD" from the EU Horizon 2020 research and innovation programme. Maria-Jesús Sobrido receives research funding from the Instituto de Salud Carlos III (grant PI17/01582).

\section{Funding}

No funding was received for the conduct of this research.

\section{Availability of data and materials}

Data sharing is not applicable to this article as no datasets were generated or analysed during the current study.

\section{Authors' contributions}

Data interpretation (MJS, PB, TdK, TK, YN, MCP, MS, CJH); drafting and revisions of manuscript (MJS, PB, TdK, TK, YN, MCP, MS, CJH). All authors approved the manuscript for submission.

\section{Ethics approval and consent to participate}

Not applicable.

\section{Consent for publication}

Not applicable.

\section{Competing interests}

PB has received travel expenses, research funding, and speaker honoraria from Actelion Pharmaceuticals Ltd. TdK has received research funding from Actelion Pharmaceuticals Ltd. TK has received research funding, travel expenses, presentation honoraria and consulting fees from Actelion Pharmaceuticals $L t d$. YN has received speech honoraria from Actelion Pharmaceuticals Ltd. and Orphan Europe, as well as travel funding from Actelion Pharmaceuticals Ltd., Shire, and Genzyme. MCP has received research grants from the Peggy Furth Fund, the National Institutes of Health [NS 65768-01], the National MS Society, Orphazyme and Actelion Pharmaceuticals Ltd., and honoraria and consulting fees from Actelion Pharmaceuticals Ltd., Alexion, Amicus, Novartis, Orphazyme, Shire HGT, Stem Cells Inc., Vtesse; stock in IntraBio; stipend and royalties from Sage Publications (Journal of Child Neurology and Child Neurology Open) and royalties from Wolters-Kluwer (Up-To-Date). MJS has received a research grant, travel expenses and speaker honoraria from Actelion Pharmaceuticals Ltd., and is a co-founder and shareholder of Genomic Consulting. MS has received travel expenses, presentation honoraria and consulting fees from Actelion Pharmaceuticals Ltd. CJH, Director of FYMCA Medical Ltd., has received consultancy fees and travel expenses from Alexion, Amicus, Biomarin, Chiesi, Inventiva, Sanofi Genzyme, and Shire, and conducted paid research on behalf of Amicus, Biomarin, Sanofi Genzyme, and Shire.

\section{Publisher's Note}

Springer Nature remains neutral with regard to jurisdictional claims in published maps and institutional affiliations.

\section{Author details \\ ${ }^{1}$ Neurogenetics Research Group, Instituto de Investigación Sanitaria, Santiago de Compostela, Spain. ${ }^{2}$ Insititute of Medical Genetics and Applied Genomics, Tübingen University, Tübingen, Germany. ${ }^{3}$ CENTOGENE AG, Rostock, Germany. ${ }^{4}$ University of Groningen, Groningen, The Netherlands. \\ ${ }^{5}$ Department of Neurology, Friedrich-Baur-Institute, University Hospital of the Ludwig-Maximilians-Universität München, Munich, Germany, German Center for Neurodegenerative Diseases (DZNE), Munich, Germany, and Munich Cluster for Systems Neurology (SyNergy), Munich, Germany. ${ }^{6}$ Department of Neurology, Reference Centre for Lysosomal Diseases (CRML), UF Neurogenetics and Metabolism, Pitié-Salpêtrière Hospital, Paris, France. ${ }^{7}$ Mayo Clinic, Rochester, MN, USA. ${ }^{8}$ Department of Neurodegenerative Diseases, Hertie-Institute for Clinical Brain Research, University of Tübingen, Tübingen, Germany. ${ }^{9}$ German Center for Neurodegenerative Diseases (DZNE), Tübingen, Germany. ${ }^{10}$ University of Pretoria, Pretoria, South Africa.}




\section{Received: 20 September 2018 Accepted: 21 December 2018} Published online: 21 January 2019

\section{References}

1. Meikle PJ, Hopwood JJ, Clague AE, Carey WF. Prevalence of lysosomal storage disorders. JAMA. 1999;281:249-54.

2. Richter T, Nestler-Parr S, Babela R, Khan ZM, Tesoro T, Molsen E, et al. Rare disease terminology and definitions-a systematic global review: report of the ISPOR rare disease special interest group. Value Health. 2015;18:906-14

3. Prevalence and incidence of rare diseases: Bibliographic data $\gg$ Orphanet report series, Rare Disease Collection 2018; 1. Available at: http://www. orpha.net/orphacom/cahiers/docs/GB/Prevalence_of_rare_diseases_by_ alphabetical_list.pdf. Accessed 13 Dec 2018.

4. Regulation (eu) no 536/2014 of the European Parliament and of the Council on Clinical Trials on Medicinal Products for Human Use. Available at: http:// eur-lex.europa.eu/legal-content/EN/TXT/PDF/?uri=CELEX:32014R0536\&qid= 1421232837997\&from=EN. Accessed 03 Sep 2018.

5. Pampols T. Inherited metabolic rare disease. Adv Exp Med Biol. 2010;686: 397-431.

6. Applegarth DA, Toone JR, Lowry RB. Incidence of inborn errors of metabolism in British Columbia, 1969-1996. Pediatrics. 2000;105:e10.

7. Nia S. Psychiatric signs and symptoms in treatable inborn errors of metabolism. J Neurol. 2014;261(Suppl 2):S559-68.

8. Bonnot O, Herrera P, Kuster A. Treatable neurometabolic diseases. Association with schizophrenia spectrum disorders. Presse Med. 2015;44: 889-97.

9. Santos-Lozano A, Villamandos Garcia D, Sanchis-Gomar F, Fiuza-Luces C, Pareja-Galeano H, Garatachea N, et al. Niemann-pick disease treatment: a systematic review of clinical trials. Ann Transl Med. 2015;3:360.

10. Wraith JE, Baumgartner MR, Bembi B, Covanis A, Levade T, Mengel E, et al. Recommendations on the diagnosis and management of Niemann-pick disease type C. Mol Genet Metab. 2009;98:152-65.

11. Patterson MC, Hendriksz CJ, Walterfang M, Sedel F, Vanier MT, Wijburg F, et al. Recommendations for the diagnosis and management of Niemannpick disease type C: an update. Mol Genet Metab. 2012;106:330-44.

12. Vanier MT. Niemann-pick disease type C. Orphanet J Rare Dis. 2010;5:16.

13. Vanier MT. Complex lipid trafficking in Niemann-pick disease type C. J Inherit Metab Dis. 2015;38:187-99.

14. Wassif CA, Cross JL, Iben J, Sanchez-Pulido L, Cougnoux A, Platt FM, et al. High incidence of unrecognized visceral/neurological late-onset Niemannpick disease, type $\mathrm{C} 1$, predicted by analysis of massively parallel sequencing data sets. Genet Med. 2016;18:41-8.

15. Hendriksz CJ, Anheim M, Bauer P, Bonnot O, Chakrapani A, Corvol JC, et al. The hidden Niemann-pick type $C$ patient: clinical niches for a rare inherited metabolic disease. Curr Med Res Opin. 2017;33:877-90.

16. Walkley SU, Davidson CD, Jacoby J, Marella PD, Ottinger EA, Austin CP, et al. Fostering collaborative research for rare genetic disease: the example of niemann-pick type C disease. Orphanet J Rare Dis. 2016;11:161

17. Vanier MT, Wenger DA, Comly ME, Rousson R, Brady RO, Pentchev PG. Niemann-pick disease group C: clinical variability and diagnosis based on defective cholesterol esterification. A collaborative study on 70 patients. Clin Genet. 1988:33:331-48.

18. Vanier MT, Latour P. Laboratory diagnosis of Niemann-pick disease type C: the filipin staining test. Methods Cell Biol. 2015;126:357-75.

19. Vanier MT. Phenotypic and genetic heterogeneity in Niemann-pick disease type C: current knowledge and practical implications. Wien Klin Wochenschr. 1997;109:68-73

20. Wijburg FA, Sedel F, Pineda M, Hendriksz CJ, Fahey M, Walterfang M, et al. Development of a suspicion index to aid diagnosis of Niemann-pick disease type C. Neurology. 2012;78:1560-7.

21. Synofzik M, Fleszar Z, Schols L, Just J, Bauer P, Torres Martin JV, et al. Identifying Niemann-pick type $C$ in early-onset ataxia: two quick clinical screening tools. J Neurol. 2016;263:1911-8

22. Pineda $M$, Mengel $E$, Jahnova $H$, Heron $B$, Imrie J, Lourenco $C M$, et al. $A$ suspicion index to aid screening of early-onset Niemann-pick disease type $C$ (NP-C). BMC Pediatr. 2016;16:107.

23. Patterson MC, Clayton P, Gissen P, Anheim M, Bauer P, Bonnot O, et al. Recommendations for the detection and diagnosis of Niemann-pick disease type C: an update. Neurology Clin Pract. 2017;7:499-511.
24. Giese AK, Mascher H, Grittner U, Eichler S, Kramp G, Lukas J, et al. A novel, highly sensitive and specific biomarker for Niemann-pick type C1 disease. Orphanet J Rare Dis. 2015;10:78.

25. Welford RW, Garzotti M, Marques Lourenco C, Mengel E, Marquardt T, Reunert J, et al. Plasma lysosphingomyelin demonstrates great potential as a diagnostic biomarker for Niemann-pick disease type $\mathrm{C}$ in a retrospective study. PLoS One. 2014;9:e114669.

26. Vanier MT, Gissen P, Bauer P, Coll MJ, Burlina A, Hendriksz CJ, et al. Diagnostic tests for Niemann-pick disease type C (NP-C): a critical review. Mol Genet Metab. 2016;118:244-54.

27. Synofzik M, Harmuth F, Stampfer M, Muller Vom Hagen J, Schols L, Bauer P. NPC1 is enriched in unexplained early onset ataxia: a targeted highthroughput screening. J Neurol. 2015;262:2557-63.

28. Hennekam RC, Biesecker LG. Next-generation sequencing demands nextgeneration phenotyping. Hum Mutat. 2012:33:884-6.

29. Andermann A, Blancquaert I, Beauchamp S, Dery V. Revisiting Wilson and Jungner in the genomic age: a review of screening criteria over the past 40 years. Bull World Health Org. 2008;86:317-9.

30. Principles and practice of screening for disease. Available at: http://apps who.int/iris/bitstream/handle/10665/37650/WHO_PHP_34.pdf;sequence=17. Accessed 03 Sep 2018

31. Bauer $P$, Balding DJ, Klunemann HH, Linden DE, Ory DS, Pineda $M$, et al. Genetic screening for Niemann-pick disease type $C$ in adults with neurological and psychiatric symptoms: findings from the ZOOM study. Hum Mol Genet. 2013;22:4349-56.

32. Schicks J, Muller Vom Hagen J, Bauer P, Beck-Wodl S, Biskup S, KragelohMann I, et al. Niemann-pick type $C$ is frequent in adult ataxia with cognitive decline and vertical gaze palsy. Neurology. 2013;80:1169-70.

33. Nanetti L. Huntington-like patients: a new phenotype for Niemann Pick type C disease? [article in Italian]. Milan, Italy: Med Point srl; 2017.

34. Topcu M, Aktas D, Oztoprak M, Mungan NO, Yuce A, Alikasifoglu M. Prospective Turkish cohort study to investigate the frequency of Niemannpick disease type $\mathrm{C}$ mutations in consanguineous families with at least one homozygous family member. Mol Diagn Ther. 2017;21:643-51.

35. Cupidi C, Frangipane F, Gallo M, Clodomiro A, Colao R, Bernardi L, et al. Role of Niemann-pick type C disease mutations in dementia. J Alzheimers Dis. 2017:55:1249-59.

36. Zech M, Nubling G, Castrop F, Jochim A, Schulte EC, Mollenhauer B, et al. Niemann-pick $C$ disease gene mutations and age-related neurodegenerative disorders. PLoS One. 2013;8:e82879.

37. Bruce CK, Smith M, Rahman F, Liu ZF, McMullan DJ, Ball S, et al. Design and validation of a metabolic disorder resequencing microarray (BRUM1). Hum Mutat. 2010;31:858-65.

38. van Egmond ME, Lugtenberg CHA, Brouwer OF, Contarino MF, Fung VSC, Heiner-Fokkema MR, et al. A post hoc study on gene panel analysis for the diagnosis of dystonia. Mov Disord. 2017;32:569-75.

39. Herbst SM, Hinreiner S, Posovszky C, Jochum F, Rödl T, Schroeder JA, et al Next generation sequencing in infantile cholestatic disorders [abstract $\mathrm{P}$ Nomin.-009]. Med Genet. 2016:120.

40. Marelli C, Guissart C, Hubsch C, Renaud M, Villemin JP, Larrieu L, et al. Miniexome coupled to read-depth based copy number variation analysis in patients with inherited ataxias. Hum Mutat. 2016

41. Pyle A, Smertenko T, Bargiela D, Griffin H, Duff J, Appleton M, et al. Exome sequencing in undiagnosed inherited and sporadic ataxias. Brain. 2015;138: 276-83.

42. McKay KE, Ruth N, Gray Z, Lloyd C, Hartley JL, Macdonald F, et al. Detection of Niemann-pick type $\mathrm{C}$ mutations in infants with liver disease using targeted next generation sequencing [abstract 163]. Mol Genet Metab. 2014;111:S76

43. Herbst SM, Schirmer S, Posovszky C, Jochum F, Rodl T, Schroeder JA, et al. Taking the next step forward - diagnosing inherited infantile cholestatic disorders with next generation sequencing. Mol Cell Probes. 2015;29:291-8.

44. Reunert J, Fobker M, Kannenberg F, Du Chesne I, Plate M, Wellhausen J, et al. Rapid diagnosis of 83 patients with Niemann pick type $C$ disease and related cholesterol transport disorders by Cholestantriol screening. EBioMedicine. 2016:4:170-5.

45. Ribas GS, Souza HM, de Mari J, Deon M, Mescka C, Saraiva-Pereira ML, et al. Selective screening of Niemann-pick type C Brazilian patients by cholestane-3beta,5alpha,6beta-triol and chitotriosidase measurements followed by filipin staining and NPC1/NPC2 gene analysis. Clin Chim Acta. 2016:459:57-62 
46. Zhang H, Wang Y, Lin N, Yang R, Qiu W, Han L, et al. Diagnosis of Niemannpick disease type $\mathrm{C}$ with 7-ketocholesterol screening followed by NPC1/ NPC2 gene mutation confirmation in Chinese patients. Orphanet J Rare Dis. 2014;9:82.

47. De Castro-Orós I, Irún P, Cebolla JJ, Rodriguez-Sureda V, Mallén M, Pueyo MJ, et al. Assessment of plasma chitotriosidase activity, CCL18/PARC concentration and NP-C suspicion index in the diagnosis of Niemann-pick disease type C: a prospective observational study. J Transl Med. 2017;15:43.

48. Sheth J, Mistri M, Sheth F, Shah R, Bavdekar A, Godbole K, et al. Burden of lysosomal storage disorders in India: experience of 387 affected children from a single diagnostic facility. JIMD Rep. 2014;12:51-63.

49. Traschutz A, Heneka MT. Screening for Niemann-pick type C disease in a memory clinic cohort. JIMD Rep Sep. 2018; [Epub porior to print].

50. Cebolla JJ, De Castro-Orós I, Irúna P, Alfonso P, López de Frutosa L, Andrade-Campos M, et al. Experience with 7-ketocholesterol and ccl18/parc as surrogated biomarkers in a series of Spanish Niemann-pick disease type C patients [abstract 45]. Mol Genet Metab. 2015;114:S29.

51. Yerushalmi B, Sokol RJ, Narkewicz MR, Smith D, Ashmead JW, Wenger DA Niemann-pick disease type $C$ in neonatal cholestasis at a north American center. J Pediatr Gastroenterol Nutr. 2002;35:44-50.

52. Hegarty R, Hadzic N, Gissen P, Dhawan A. Inherited metabolic disorders presenting as acute liver failure in newborns and young children: King's college hospital experience. Eur J Pediatr. 2015;174:1387-92.

53. Verity C, Winstone AM, Stellitano L, Will R, Nicoll A. The epidemiology of progressive intellectual and neurological deterioration in childhood. Arch Dis Child. 2010;95:361-4.

54. Winstone AM, Stellitano LA, Verity CM. Niemann-pick type $C$ as a cause of progressive intellectual and neurological deterioration in childhood. Dev Med Child Neurol. 2017;59:965-72.

55. Corry PC. Consanguinity and prevalence patterns of inherited disease in the UK Pakistani community. Hum Hered. 2014;77:207-16.

56. Lisi EC, McCandless SE. Newborn screening for lysosomal storage disorders: views of genetic healthcare providers. J Genet Counsel. 2016;25:373-84.

57. Lisi EC, Gillespie S, Laney D, Ali N. Patients' perspectives on newborn screening for later-onset lysosomal storage diseases. Mol Genet Metab. 2016;119:109-14.

58. Patterson MC, Mengel E, Wijburg FA, Muller A, Schwierin B, Drevon $\mathrm{H}$, et al. Disease and patient characteristics in NP-C patients: findings from an international disease registry. Orphanet J Rare Dis. 2013;8:12.

59. Imrie J, Heptinstall L, Knight S, Strong K. Observational cohort study of the natural history of Niemann-pick disease type $C$ in the UK: a 5-year update from the UK clinical database. BMC Neurol. 2015;15:257.

60. Pinto $\mathrm{R}$, Caseiro $\mathrm{C}$, Lemos $\mathrm{M}$, Lopes $\mathrm{L}$, Fontes $\mathrm{A}$, Ribeiro $\mathrm{H}$, et al. Prevalence of lysosomal storage diseases in Portugal. Eur J Hum Genet. 2004;12:87-92

61. Polo G, Burlina A, Furlan F, Kolamunnage T, Cananzi M, Giordano L, et al. High level of oxysterols in neonatal cholestasis: a pitfall in analysis of biochemical markers for Niemann-pick type C disease. Clin Chem Lab Med. 2016:54:1221-9.

62. Geberhiwot T, Moro A, Dardis A, Ramaswami U, Sirrs S, Marfa MP, et al. Consensus clinical management guidelines for Niemann-pick disease type C. Orphanet J Rare Dis. 2018;13:50.

63. de Koning TJ, Jongbloed JD, Sikkema-Raddatz B, Sinke RJ. Targeted next-generation sequencing panels for monogenetic disorders in clinical diagnostics: the opportunities and challenges. Exp Rev Mol Diag. 2015; 15:61-70.

64. Alobaidy $\mathrm{H}$. Recent advances in the diagnosis and treatment of niemannpick disease type $C$ in children: a guide to early diagnosis for the general pediatrician. Int J Pediatr. 2015;2015:816593.

65. Wittmann J, Karg E, Turi S, Legnini E, Wittmann G, Giese AK, et al. Newborn screening for lysosomal storage disorders in Hungary. JIMD Rep. 2012;6:117-25.

66. Garg U, Dasouki M. Expanded newborn screening of inherited metabolic disorders by tandem mass spectrometry: clinical and laboratory aspects. Clin Biochem. 2006;39:315-32.

67. van Karnebeek CD, Mohammadi T, Tsao N, Sinclair G, Sirrs S, Stockler S, et al. Health economic evaluation of plasma oxysterol screening in the diagnosis of Niemann-pick type C disease among intellectually disabled using discrete event simulation. Mol Genet Metab. 2015;114:226-32.

68. Wortmann SB, Vaz FM, Gardeitchik T, Vissers LE, Renkema GH, SchuursHoeijmakers $\mathrm{JH}$, et al. Mutations in the phospholipid remodeling gene
SERAC1 impair mitochondrial function and intracellular cholesterol trafficking and cause dystonia and deafness. Nature Genet. 2012;44:797-802.

69. Roeben B, Schule R, Ruf S, Bender B, Alhaddad B, Benkert T, et al. SERAC1 deficiency causes complicated HSP: evidence from a novel splice mutation in a large family. J Med Genet. 2018;55:39-47.

70. Mavridou I, Cozar M, Douzgou S, Xaidara A, Lianou D, Vanier MT, et al. Niemann-pick type C disease: a novel NPC1 mutation segregating in a Greek island. Clin Genet. 2014;85:543-7.
Ready to submit your research? Choose BMC and benefit from:

- fast, convenient online submission

- thorough peer review by experienced researchers in your field

- rapid publication on acceptance

- support for research data, including large and complex data types

- gold Open Access which fosters wider collaboration and increased citations

- maximum visibility for your research: over $100 \mathrm{M}$ website views per year

At BMC, research is always in progress.

Learn more biomedcentral.com/submissions 